\section{Production Processes for Creating Educational Videos}

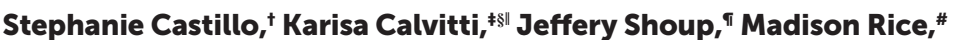 \\ Helen Lubbock, ${ }^{\circledR * *}$ and Kendra H. Oliver ${ }^{\dagger * * *+t *}$ \\ ${ }^{\dagger}$ Communication of Science and Technology Program, College of Arts and Science; ${ }^{\circledR}$ Department \\ of Teaching and Learning, Peabody College; ${ }^{* *}$ The Wond'ry, "tDepartment of Pharmacology, and \\ 'Basic Sciences, School of Medicine, Vanderbilt University, Nashville, TN 37232; "Department of \\ Pathology, Microbiology, and Immunology and \$Vanderbilt Institute of Infection, Immunology, and \\ Inflammation, Vanderbilt University Medical Center, Nashville, TN 37232; "Master of Liberal Arts \\ and Science Program, College of Arts and Science; "Biomedical Visualization, University of Illinois, \\ Chicago, IL 60607
}

\begin{abstract}
Asynchronous video-based educational resources allow for increased course material engagement. In today's climate, educators are encouraged to create videos for online instruction but are typically given limited production guidance. Few formal resources exist to guide educators for high-quality video production in a non-studio setting. This article is a how-to guide for producing videos using widely available primary resources through three steps: preproduction, production, and postproduction. During preproduction, educators consider style and project scope, including the "what, how, and why" of the content. For production, we have provided information on the set, light, sounds, and video equipment needed for optimizing video production in a non-studio setting. Finally, during postproduction, the educator considers how to combine and edit the video as well as organize content. Overall, this article is an approachable guide to help educators begin their low-budget video-production journeys.
\end{abstract}

\section{INTRODUCTION}

While many biomedical science educators are aware of the benefits of using video, there is a lack of resources that describe and summarize the video-production process. Decades of research have demonstrated that video can be used to enhance learning. The combination of verbal instruction with visual images significantly increases recall and retention in students (Mayer, 2014; Supplemental Figure 1). The video content's impact can also be enhanced through approaches based on multimedia learning principles (Issa et al., 2011). Video has been used in many educational scenarios, including traditional lectures, guided discussions, and self-observation sessions (Pinsky and Wipf, 2000). Furthermore, video has been used increasingly for coaching, mentoring, and professional development in educational settings (Nemirovsky and Galvis, 2004). In contrast to the plentiful amount of research supporting video use, few accessible resources are available to support educators as producers.

Educators have been asked to take on new roles as universities rapidly transitioned education online in response to the COVID-19 global pandemic. While the transition from in-person lectures to videos may be intimidating, it is also an opportunity for instructors to develop new skill sets. Video, defined as electronic recordings that contain both audio and visual elements, has been used for science education and communication for decades (McGarr, 2009; Rajadell and Garriga-Garzón, 2017). The shift toward online learning has challenged faculty to adopt video instruction into their teaching practices in either a live setting (synchronous) or through prerecorded videos (asynchronous). By learning how to produce video content, educators can build flexibility in teaching practices. Video has already become a valuable tool to support
Daron Barnard, Monitoring Editor

Submitted Jun 18, 2020; Revised Mar 22, 2021; Accepted Mar 24, 2021

CBE Life Sci Educ June 1, 2021 20:es7

DOI:10.1187/cbe.20-06-0120

*Address correspondence to: Kendra H. Oliver (kendra.h.oliver@vanderbilt.edu).

(c) 2021 S. Castillo et al. CBE-Life Sciences Education $\odot 2021$ The American Society for Cell Biology. This article is distributed by The American Society for Cell Biology under license from the author(s). It is available to the public under an Attribution-Noncommercial-Share Alike 3.0 Unported Creative Commons License (http://creativecommons.org/licenses/ by-nc-sa/3.0)

"ASCB $®$ " and "The American Society for Cell Biology $\circledR^{\circledR}$ are registered trademarks of The American Society for Cell Biology. 
adaptable and self-paced learning. However, students interact with video beyond the classroom and have high expectations for visual and audio quality. Therefore, to make compelling videos, educators should be supported and empowered as producers of video content.

The modern ubiquity of phones with cameras and other portable devices allows most people to easily watch and create videos. Many educators have transitioned to virtual teaching and are becoming comfortable with synchronous Web conferencing using Zoom, Skype, Microsoft Teams, and other platforms. Prerecorded online lectures in these programs can be used as asynchronous videos, supporting classroom accessibility by allowing students to rewatch lectures at any time (Clark et al., 2015). Some educators have even attempted to fulfill both synchronous and asynchronous needs by recording Web-based lectures. While recording a Web-based session does fill the role of content acquisition, it is not ideal for several reasons. For one, the quality is generally poor and can distract later audiences. In addition, Web-based lectures are designed for the synchronous audience and, most likely, are suboptimal to the learning experience of the asynchronous viewer. As compared with recorded lectures, produced videos can deploy the multimedia learning theory supported by Mayer (2014), which may not be a consideration for a standard synchronous videoconferencing lecture (Mayer and Pilegard, 2014). Intentional production of content has been shown to increase the impact on learning outcomes with increased motivation, satisfaction, and perception toward the material (Um et al., 2012). These studies emphasize style, format, and quality as essential in multimedia educational learning materials. To address this goal within low-budget settings, additional resources are needed to help educators produce quality videos in at-home environments.

At first glance, the process of video production could be daunting to some educators, as there are many different styles of videos to consider creating. Educators must also familiarize themselves with the technology and editing software and develop an overall production process (Petrosino and Koehler, 2007). There is a wide range of equipment that may be confusing to someone unfamiliar with video production. Notably, with many working from home, educators need to establish simple, low-budget at-home studios. Thankfully, the evolution of video technology has made it easier and cheaper to record high-quality videos by implementing minor design decisions.

We have created this resource to support beginner educator-producers using their phones and at-home setups to minimize the barriers to producing high-quality videos. In this paper, we present workflows to guide educators in making several types of videos for their courses and producing simple, effective, and engaging videos with a budget-friendly, at-home setup. Educators begin this process starting with preproduction (lesson design and storyboarding), move through production (setup, styles, and lecture recording), and end in postproduction (video editing). We also cover production theory extensively before walking through production workflow and the description of the video styles. Covering the theory behind producing videos will help educators understand the mechanics of producing high-quality videos. Herein, we provide a production process for creating asynchronous video content through using an at-home studio setup and a phone with a camera. The main product is a workflow (Figure 1) for the educator to begin to take on the producer's role in online video content production.

\section{PREPRODUCTION}

By far, the most significant amount of time in the video-production process is spent in preproduction. Preproduction refers to any of the planning stage activities before filming and has been well canvassed in the literature (Jenkinson, 2017). This critical stage is commonly underestimated and underutilized when it comes to crafting a video for online learning (Chang and Hirsch, 1994; Currie, 2003; Corbally, 2005). Preproduction involves creating a design that uses the strengths of video production and optimizes them for the audience's learning capabilities (Jenkinson, 2017). Lesson design and storyboarding are key components of preproduction. A storyboard is a visual representation of a video's timeline (Orr et al., 1994). It breaks down the video timeline into individual panels containing all instructions necessary for the audio script and the corresponding visual elements such as on-screen text, animations, or the recorded video (McGill, 2017). Storyboarding is specific to video production, whereas lesson planning typically occurs in teaching environments. For this purpose, the educator may want to specifically refer to this step as storyboarding to distinguish it theoretically from lesson planning.

In storyboarding, the specific objectives for the video, as well as the script, determine the optimal video structure and style. Assuming lessons have already been designed, storyboarding should be a distinct process crafted for video medium (Orr et al., 1994; McGill, 2017). The storyboard eventually becomes the guiding design document that outlines the production process. This document serves as a guide that can be reviewed and approved by any co-instructors, content experts, and instructional designers, administrators, or other stakeholders before commencing production. Furthermore, a storyboard allows for increased style and format consistency across videos. Consistent formatting and repeated graphics help reduce cognitive load for the student (Orr et al., 1994; Paas and Sweller, 2014; Brame, 2016; McGill, 2017). The goal of preproduction is for educators to walk away with a detailed storyboard that will guide the production workflow. Here, we break down the storyboard into three sections: contextualizing videos within a course, clarifying learning objectives, and video mechanics. Production efforts that are intentionally designed will help guide the educator-producer during production and postproduction.

\section{Video Contextualization}

From a broad perspective, the educator-producer should consider diversified instructional approaches such as the implementation of video within the course plan (Pritchard, 2018; Dunn and Rundle, 2000; Griggs, n.d.). To decide when and where to include videos, how many videos to produce, or what topics the videos will cover, educators can examine and depend on their syllabi. The syllabus should be a user-friendly and meaningful document that logically directs the faculty member and student through the course content (Huang et al., 2019). While reviewing or revising a syllabus, educators should make sure the addition of a video and each video's assigned fit addresses the course learning objective. Consider content mapping, which refers to delivering the right content, to the right people, at the right time. In particular, it is critical to think 


\section{Production बheskllist for beginners}

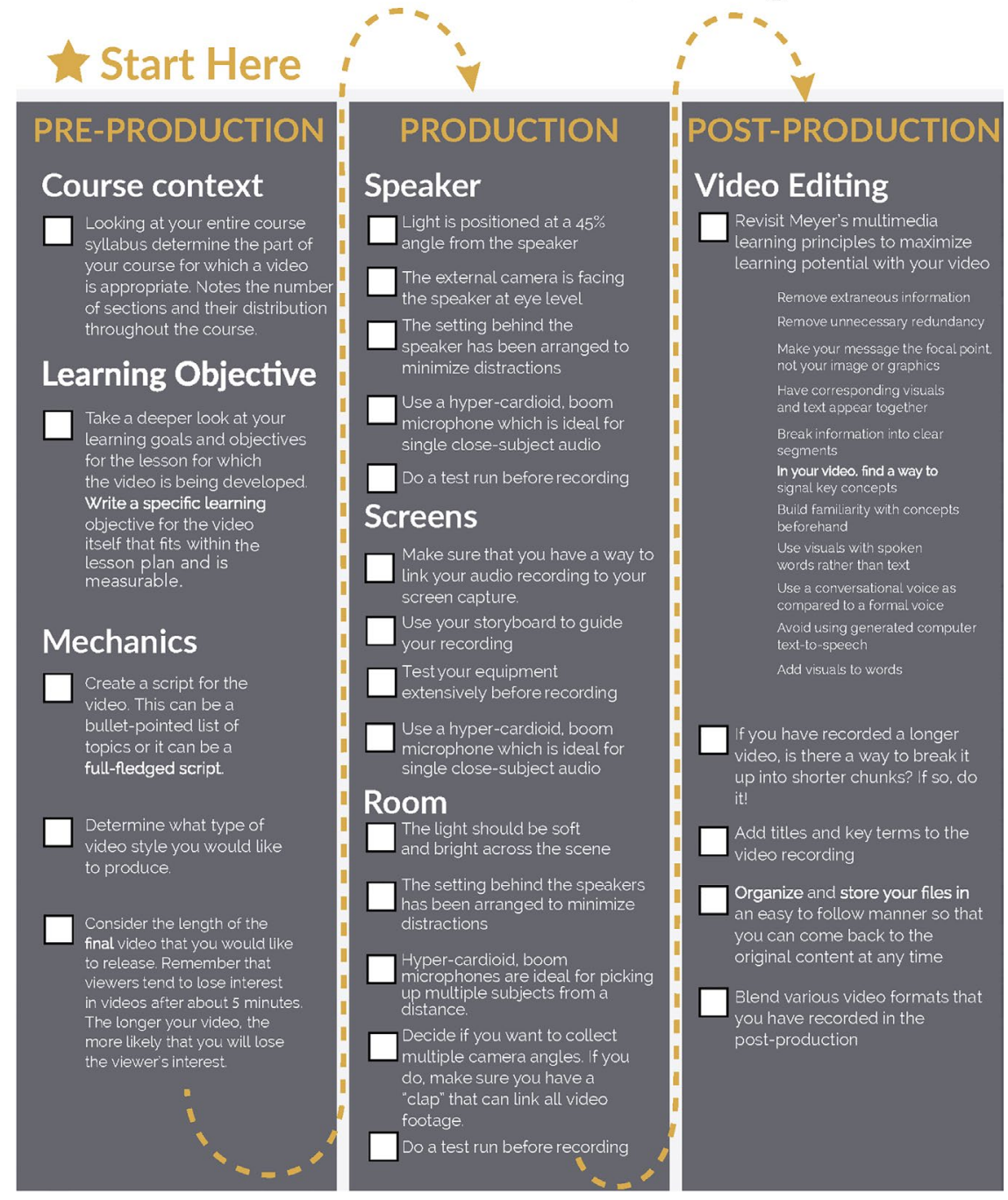

FIGURE 1. Production checklist. To provide a streamlined guide for science educators and scientists to embark on their production process, we have created a production checklist that outlines the preproduction, production and postproduction processes. This checklist can be downloaded for a clickable guide for educators (https://vanderbilt.box.com/s/ twtwh5tz9uc8jiyt65ixnuffa9vg52dm). which students acquire, process, and maintain knowledge. Connectivity is the relationship between student and material and the relationship between student and educator. One way to hit all three aims in online classrooms is including asynchronous videos in addition to synchronous videos (Clark et al., 2015). Meaningful interactivity promotes student learning (Cairncross and Mannion, 2001; Anderson et al., 2008; Antonietti et al., 2015). For example, holding discussion during synchronous lectures and then use asynchronous videos to further explain or demonstrate the context of the lesson, or vice versa. Establishing the best connectivity is dependent on which video style is chosen, which we go over in depth in Production Guides section.

\section{Learning Objectives}

Before deciding which video style to pursue, it is essential to clarify the learning objective. Learning objectives are brief statements that describe what a student should be able to do or is expected to know at the end of a lesson or, in this case, after watching a video (Black and Wiliam, 1998). Prior knowledge of the video topic may vary between different audiences, so content and learning objectives are essential (Johnson and Hertig, 2014). In many cases, learning objectives build upon each other toward reaching an overall learning goal. As compared with a learning goal, a learning objective is measurable. The objectives must be written in ways that allow the educator to measure whether or not students have achieved them accurately. As an educator begins to think about the learning objective for an educational video, the educator must define the learning objective in a measurable format so that the video's impact can be assessed through the course. about how, when, and where the student is engaging with the video content. After reflecting on the syllabus and mapping where a video will be included, producers should next consider the video as a relationship-building opportunity with the virtual student.

Within a virtual education space, educators and students may never have an in-person meeting or conversation. Strategic online instructional design and emotional connectivity created by the video style develop a better relationship with the student (Mayer and Estrella, 2014). To build this relationship, context, learning modes, and connectivity should all be examined during the preproduction process. Context is what the video will discuss, which is determined by your lesson plan and syllabus. Learning modes are the methods or ways in

\section{Video Mechanics}

The third step that occurs in preproduction is evaluating the mechanisms of how the video will be produced. This portion consists of three aspects for intentional video design: script, style, and length. While rarely used for traditional teaching, scripts for online asynchronous content allow the educator to review material, minimize extraneous information, and clarify learning objectives. Scripts should be developed by looking at slide order, content included on those slides, and other information relevant to each slide that is not visually depicted. Educator who are more comfortable reading lecture notes can use voice-to-text software to facilitate this process. While a script can contain each and every word that will be spoken, a script can also be a general guideline to help stay on track 
when filming-essentially creating an outline with talking points and what needs to be addressed for each slide or topic covered. However, if a verbatim script is created, the document can be used to produce closed captioning to promote video accessibility.

Next, the educator should consider various video styles and how each style complements each section of the script. Choe et al. (2019) evaluated eight different video styles designed to deliver standardized content in the life sciences. Here, because of the audience and resource limitations, we have simplified these styles into categories of Speaker, Screen, and Room. These styles will be covered in-depth in the Production Guides portion of this document. Choosing the optimal video style depends on the lecture content, learning objectives, and how educators like to teach their courses. When deciding on a style, educators should consider interactivity and engagement with the learner (refer back to Video Contextualization). No one style works for all educators, and educators should consider their individual strengths when choosing a video style. Additionally, it might be worthwhile to employ a combination of style. Be aware that if multiple styles are used in one video, more postproduction is required to connect the distinct video styles.

The last mechanics consideration is the length of the video. Research supports short videos divided into sections or segments, as compared with a 40-minute video of a traditional lecture (Brame, 2016). As video length increases, students lose focus and become less engaged with the material (Farley et al., 2013). If it is easier for the educator, entire lectures may be recorded in one sitting and split later during postproduction, but this should be planned beforehand. One way to punctuate a single long recording is by incorporating questions or moments of reflection within the lecture. Breaking up the video with questions can guide student learning and help transition into the next video or assignment. In addition, incorporating moments of reflection through rhetorical questions or recap statements can prompt students to pause and consider whether they have internalized the video's learnings or should rewatch the video. While an intricate video may be more exciting to watch, unnecessary elements can also add an extraneous load that may limit the video's ultimate effectiveness (Corbally, 2005; Brame, 2016). To prevent this, educators should continuously revisit their scripts in light of the learning objectives and determine whether the style chosen best represents the material.

\section{PRODUCTION THEORY}

Production refers to all activities that involve the recording of audiovisual material. When starting production, it is essential to recognize equipment and resource limitations. For high-production video styles, a full recording studio may be required; this could include soundproofing to eliminate echo and outside noise, a raised floor to minimize vibrations that may cause audio interference, and overhead ceiling-mounted lights to provide additional lighting options. A fully equipped studio may include teleprompters and monitors for the presenter as well. With the assumption that most educators do not have access to a full studio setup, we will be considering production for an at-home studio setup using a phone camera.

As the educator takes on the role of the producer, it is important to assess the audio and visual goals of the videos and their feasibility before starting production. Thinking about the limitations of production can help create realistic expectations for the final products. High-quality videos can still be achieved through budget-friendly setups if educators apply production theory to their videos. Four key elements must be considered for optimal video production: light, sound, video, and set. For the sake of simplicity, we will refer to the phone camera as "the camera" and will specifically note when we are referring to a stand-alone camera (including single-lens reflex camera [SLR], digital-SLR [DSLR], or camcorder). Please note that many of these theories can apply to both a phone camera and a professional camera.

\section{Lighting}

Lighting is key to making high-quality videos. With the right lighting, it is difficult for viewers to discern between a phone camera and a professional camera. Lighting should generally be placed at a $45^{\circ}$ angle down at the subject from above (Figure $2 \mathrm{~A})$. It should also be placed off-center, $\sim 45^{\circ}$ left or right of the subject, and out of frame. The inverse-square law applies to light, meaning that the closer the subject is to the light source, the more drastic the change in light level or intensity will be on the subject. As the light source is moved farther away from the subject, the drop in light level based on the distance from the light source is dramatically reduced, allowing softer diffused light to hit the subject. Additionally, the larger the light source, the softer and more flattering the light will be on the subject. If no lighting budget is available, a window can be used. Have the camera placed such that the window is $\sim 45^{\circ}$ off-axis. If the light seems harsh, put some translucent drapes across the window or sit farther away. While a window might be a low-budget option, keep in mind that it will be difficult to build consistency with natural lighting across multiple filming days.

When additional speakers or objects are added to the frame, it is particularly important to be mindful of the lighting. For a recording that includes multiple speakers or objects, larger light sources can help ensure the lighting is bright, soft, and even. If a light source is not available, perform the demonstration or the interview straight on-axis with the window. This will give even light across both subjects and objects. In this case, the light should enter directly behind the camera, leading to even lighting across the scene. For those who are interested in purchasing equipment, two equally large and bright light sources on either side of the camera at a $45^{\circ}$ angle above and to the side of both subjects would be suggested, similar to the structure of an individual subject recording.

Another consideration with light is temperature. White balance is the process of having objects that appear white in reality rendered white in your video. The light is measured by temperature on the Kelvin scale. Typically, Kelvin temperatures for commercial and residential lighting applications fall somewhere on a scale from $2000 \mathrm{~K}$ to $6500 \mathrm{~K} .3200 \mathrm{~K}$ is the temperature at which tungsten burns, and often the temperature of soft interior incandescent lighting, while $5500 \mathrm{~K}$ is the temperature of sunlight. It is important to have a single temperature illuminating a subject's skin. For example, room lights and sunlight should not enter the frame at the same time, and any light fixtures intended to remain in the frame should be as dim as possible. It is often advisable to turn off all interior lighting (room lights) to avoid mixed light. 


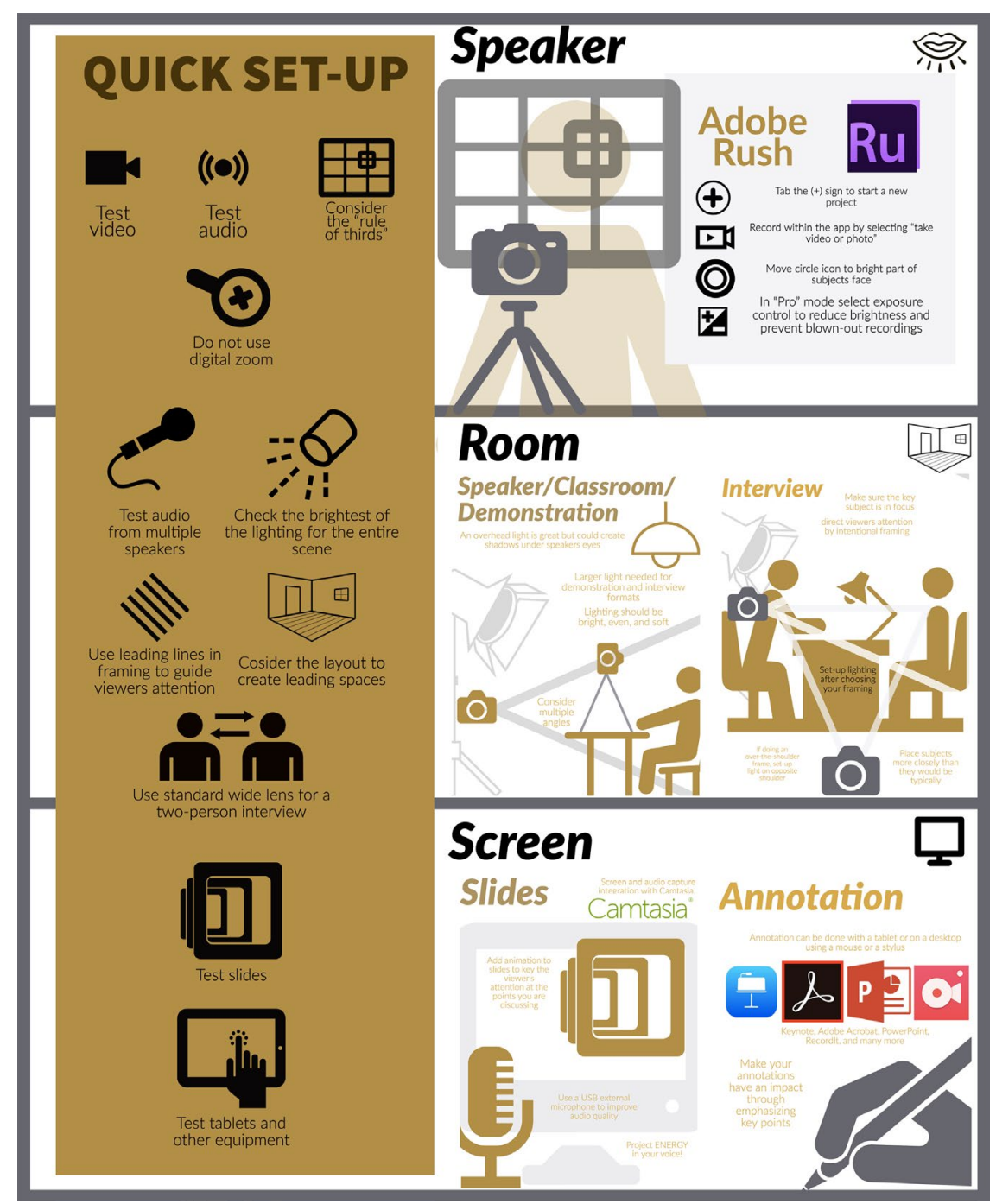

FIGURE 2. Production guides for speaker, room, and screen recordings. A quick setup list can be found on the left side of the graphic. The items listed in the quick guide are arranged vertically based on their relationship to the format type (speaker, room, screen). Within each format, production suggestions are shown within the infographic. Within "Speaker," the steps for recording using Adobe Rush are shown in addition to the rule of thirds for a single speaker using a graphic. In "Room," more detailed recommendations for the speaker, classroom, and demonstration formats are provided. Additional recommendations for an in-person interview-style format are also provided in this section. Finally, within the "Screen" recording section, slides and annotation suggestions are provided. The icon on the upper right-hand side of each format is correlated to the various formats outlined by Choe et al. (2019) in Figure 4.

\section{Sound}

Poor-quality videos with clear sound are more likely to be accepted by an audience than a high-quality video with poor audio. To record crisp and clear audio, it helps to record in a quiet room to achieve as large a signal-to-noise ratio as possible. The signal-to-noise ratio is based on the difference in the volume of the subject and the background noise that cannot be controlled or removed. The larger the difference between the audio of the speaker versus background noise, the larger the signal-to-noise ratio is, resulting in better audio quality. To achieve low background sound, turn off air conditioners, fans, and buzzing cell phones. Set up away from noisy windows or appliances like refrigerators. If loud background noise cannot be avoided, position the camera to avoid collecting the sound or consider using an external microphone, as discussed below.

A microphone diaphragm is the thin membrane that vibrates with sound pressure levels and produces the electrical signal that becomes the analog audio wave. The size of the diaphragm regulates some of the basic characteristics of the resulting wave. Large-diaphragm microphones tend to have better low-frequency reproduction. These types of microphones are often used in radio, voice-overs, vocal recordings, and podcasts, where sound is key and having a large microphone in front of the person speaking is not an issue. The positioning of the microphone to reject background noise is based on its polar pattern. The polar pattern of a microphone is a graph of the microphone's sensitivity to picking up sound arriving around the central axis of the microphone capsule. Polar pattern plots show the sensitivity of the microphone across various angles tested at different frequencies, depicted with separate lines for each frequency tested (Figure 2B).

There are three types of polar patterns for capturing audio for video: cardioid, omnidirectional, and hyper-cardioid. Cardioid polar pattern microphones are ideal for picking up one or more subjects from a close to midrange distance. Microphones with this pattern are a good option for recording interviews. The microphone can be placed between the two subjects pointed down at the center of the conversation with the back facing the nosiest part of the space. This will capture even sound while rejecting unwanted noise. An omnidirectional pattern is often found in lavalier microphones; this pattern makes them able to pick up audio from all directions, which makes placement on the subject much easier. Hyper-cardioid patterns are often found in boom microphones and work well for single close-subject audio or picking up multiple subjects from a distance.

While phone cameras can be used to collect high-quality visual recordings, external devices are generally needed to improve the audio quality. There are multiple approaches to collecting high-quality sound. A lavalier microphone, sometimes referred to as a lapel microphone, might be useful but can present its own challenges. A lavalier microphone could have unintentional periods in which unwanted sounds, like clothing ruffle, are collected or when audio is not collected at all. Lavalier microphones are also susceptible to poor placement, which can 
lead to poor audio quality. The lavalier microphone capsule should be omnidirectional, meaning that it is receiving signals from or transmitting in all directions. The lavalier microphone should be placed midsternum center on the chest. If the subject is within 3 feet of the camera, a boom or hyper-cardioid microphone may be best. A boom microphone will sound natural and requires less troubleshooting to assure quality. The boom microphone should be out of the video frame and be no more than 1 to 3 feet from the subject. If you are conducting an interview with a soft-spoken guest, each person in the video can be provided a lavalier microphone. There are several options compatible with a cell phone, but the distance from the camera should be considered. An extension might be necessary. The boom should be pointing at the subject's chest in the direction the person is most often speaking. Additionally, it is important to note that all microphone types can be connected to a cell phone. Finally, if your audio-collection and video-collection mechanisms are not synchronized, use a "clap" to help synchronize the audio and video in postproduction. This can either be a hand clap, a film slate, or some other event that can coordinate visual and audio cues.

\section{Framing}

Framing, or composing the visual content of a series of frames as seen from a single point of view, is essential to direct the viewers' attention. When positioning speakers or objects in the frame, use the rule of thirds. This grid breaks up the frame into three equal parts, both vertically and horizontally. The main subject of the frame should be placed on either the left or right vertical line with the eyes where the top horizontal line intersects the vertical line, or in the center of the frame. If the subject is facing the interviewer or otherwise obstructing the key light to the left of the camera, the subject should be placed on the right vertical line. This will provide leading space or space in the subject's gazing direction that fosters a pleasing and clear image for the viewer. Even if other elements are in the frame, organizing frame composition using the rule of thirds will direct the viewer's attention (Figure $2 \mathrm{C}$ ).

Another key consideration for proper framing is focal length, which is the distance between the lens and the point where the in-focus image is formed inside the camera. Changes in the focal length impact the angle of view for the camera. Also, the same lens on different cameras can also result in different angles of views, because the light sensors may not be identical. To make comparisons across cameras, the $50 \mathrm{~mm}$ lens is considered the standard full-frame format for a stand-alone camera. Lenses above $50 \mathrm{~mm}$ are in the "tele" zone that compresses perspective, whereas lenses less than $50 \mathrm{~mm}$ are within the "wide" zone that exaggerates features. With a phone camera, these measurements do not directly correspond to those on stand-alone cameras (SLR, DSLR, or camcorder) and must be calibrated. For example, the wide-angle lens on the iPhoneX has a focal length of $4.25 \mathrm{~mm}$, which is a $26 \mathrm{~mm}$ equivalent. The telephoto lens on the iPhoneX is $6 \mathrm{~mm}$, equivalent to a $52 \mathrm{~mm}$ full-frame camera. The rear-facing camera on the iPhone, now called the TrueDepth camera, has a $2.87 \mathrm{~mm}$ focal length.

For a one-person shot, or for an interviewee framed over the interviewer's shoulder, consider a telephoto effect to separate the subject from the background. Make sure not use the digital zoom, because this will reduce the resolution of the video captured. Instead, use the $2 \times$ lens or other options that may be available based on the phone camera. Many phones have two to three lens options, and more details can be found in each phone model's user manual. When in doubt, use the wide-angle option. Wide-angle filming options, or a lens that allows more of the scene to be included in the video frame, generally have a larger aperture size, letting in more light and producing a sharper image with a natural focal length. If the decision is made to use a wide-frame filling approach, it is also possible to crop the frame during postproduction to make the video more interesting and dynamic.

\section{Video}

Video quality impacts the utility and quality of your video. In terms of equipment, the cameras in phones have become quite advanced, with several features that have improved the quality of images, including optical image stabilization, larger sensors, bright lenses, and optical zoom. Since the advent of widescreen TV (aspect ratio of 16:9), the term "HD video resolution," which refers to a video of higher resolution, typically greater than 720 $\times 480$ pixels, has been used to refer to the measurement of pixels. A pixel (also referred to as pel or picture element) is the smallest addressable element on a display element of a screen. The "standard" HD resolution is 1080 , which refers to a resolution of $1920 \times 1080$. However, $4 \mathrm{k}$ is quickly becoming the new standard for video distribution, as data speed and video compression improve. When referencing a 16:9 aspect ratio, $4 \mathrm{k}$ resolution is $4096 \times 2304$. This resolution is often referred to as "ultra high definition." Most modern phones are capable of $4 \mathrm{k}$ video or higher. Also keep in mind that front-facing cameras for phones and laptops typically have relatively low resolution, as they are designed for functionality rather than image quality (Figure 2D).

A few camera functions that affect video quality retain their historical names but are now performed through digital processing. For example, digital cameras no longer contain mechanical shutters, but shutter-speed values can be adjusted to mimic how film cameras allow light to expose the film. Shutter speed is measured in fractions of 1 second (e.g., a shutter speed of $1 / 50$ would expose the film for 1/50th of 1 second). Additionally, the aperture is not an adjustment that occurs physically for built-in phone cameras. Instead, the exposure setting automatically adjusts based on internal algorithms of the device designed to optimize video quality. However, it is likely that the videographer will need to adjust these settings to avoid underexposure or overexposure based on a number of setting factors. Ultimately, it is best to err on the side of underexposure versus overexposure.

\section{Set}

Considering the set is essential to minimize distracting background items. It is key to consider consistency and intentionality for all video elements, including the background. One key aspect of building a set is to ensure minimal distractions behind the subject that would pull the viewers' attention away from the purpose of the video. Often, this means that simpler is better. A plain, neutral-colored wall with a simple plant or background object to break up the wall might be sufficient. Be mindful of objects like lamps and plants. A poorly placed plant behind the subject could make it look like the plant is growing from the top 
of the speaker's head. If curating the set is not possible, hanging a fabric backdrop can also work well. Blurring the background can also minimize details and add focus on the subject. A blurry background is achieved by wider apertures with longer focal lengths combined with increasing the distance between the subject and the background. To attempt this effect on a phone camera, click on the subject of the frame so that it is in focus and separated from the background. This is a great option when busy backgrounds are unavoidable (Figure 2E).

\section{Editing Software}

There are several apps available for making high-quality recordings using a phone. We will be focusing on the Adobe Premiere Rush app, because it is free and available for iOS, Android, and Windows (Supplemental Video 1). It is fairly approachable for users compared with the more complicated Adobe Premiere Pro video-editing software. Ideally, as the educator-producer's skill increases, these files can be transitioned to Adobe Premiere for more sophisticated postproduction editing. Using Adobe Rush, it is possible to record content directly into a project within the application. Properly setting the exposure is an essential aspect of creating a high-quality video. The exposure circle must be placed over the brightest part of the skin. ISO is like a volume knob for light; higher ISO values digitally increase the sensitivity of the light sensor. If the light input becomes too overwhelming, video footage appears overexposed and is unusable (Figure $2 \mathrm{~F}$ ). If editing on a computer is preferred, we recommend Camtasia as a screen-capturing and video-editing program (Figure 2G; Supplemental Video 2), but there are also free alternatives, including iMovie and OpenShot. We feel that Camtasia, although it costs more than $\$ 200$, is a great middle ground resembling the simplicity of the Adobe Rush app with the editing capabilities of Adobe Premiere Pro. The built-in graphics, animation, and transition features facilitate editing for educators to have more control in tailoring and enhancing their videos. Whichever editing software you select, it is important to spend time familiarizing yourself with the options available to optimize your editing process.

\section{PRODUCTION WORKFLOW}

The production guides presented here mainly employ Adobe Premiere Rush, a free, simple, and accessible software that can prepare educator-producers for more advanced software such as Adobe Premiere Pro. We also discuss Camtasia, which is a proprietary screen recorder and video editor with a free trial currently available. There are many additional recording software options to choose from. More extensive and regularly updated lists are provided on the website resource that we have created (www.scivid.online). Please refer back to Supplemental Videos 1 and 2 to learn more about the similarities and differences of Adobe Premiere Rush and Camtasia.

\section{Adobe Premiere Rush}

For the scope of this Essay, we limit this workflow discussion to a recording using Adobe Premiere Rush and the built-in phone camera (Figure 3). When the Adobe Rush app is opened, a plus [+] sign can be seen at the bottom; this will create a new project. There is the option to start with precaptured media (camera roll) or to start taking a photo or recording a video right from the app. Selecting "take video or photo" will open the camera, oftentimes on auto mode. Move the circle icon around the screen to the brightest part of the subject's face. In the Pro mode of Adobe Premiere Rush, the default setting is still set to automatically govern the camera. This is where the camera analyzes the image and selects the appropriate settings for the scene. However, from this interface, it is possible to select the exposure compensation. While the exposure might be ideally set using the auto-selection, sometimes it may not be properly selecting the subject's skin and may need adjustments. Use +/- exposure control to increase or, more often, decrease the exposure compensation until the subjects are bright but not overexposed, or allowing in too much light that reduces the quality of the video. Then select the exposure adjustment (lens aperture icon to the left of the exposure) and make sure the automatic setting is turned off. This will lock in the exposure settings, and the phone will be ready to begin recording.

\section{PRODUCTION GUIDES}

Choe et al. (2019) outlined eight different types of video styles used in an educational setting. These video styles include classroom, weatherman, demo, learning glass, pen tablet, talking head, and slides on/off. These classifications help set the terminology educators can use to refer to various video styles (Choe et al., 2019; Figure 4).

\section{Speaker Style}

The speaker style (Supplemental Videos 3 and 4) has become a popular approach. This style typically captures the educator in full-frame from the mid-shoulders up, with slight space above the head. Video of the speaker can be used alone or in conjunction with slides and pen tablet as a lower-third. For this style, a phone camera, a microphone, and a small tripod or stand are required equipment. Position the phone horizontally on the tripod. Then, place the phone and tripod such that the educator is at eye level with the camera and in good lighting following the aforementioned theory. If the educator will refer to notes or lecture slides while recording, position the references out of frame. However, the reference material should be close enough that the educator still appears to be facing the camera when reading the material. It is important to test this extensively before recording. For the educator to appear natural and engaging, having consistent "eye contact" with the camera gives the illusion the educator is speaking directly to the student. Ideally, notes should not be read verbatim, and a calm conversational tone should be used. Although the educator may feel restricted, uncomfortable, or reserved speaking in front of a camera, using positive body language and facial expressions helps to convey interest and retain the student's attention.

\section{Screen Style}

Video formats that capture screen content, such as slides or pen tablet, are straightforward options for beginners (Supplemental Videos 5 and 6). These approaches can be very forgiving in that they are screen-captured and do not require considerations for lighting and camera focus. For this type of video style, a USB microphone and any screencasting software are required materials. Because the educator is not on camera, it is possible to use notes without considering how their placement affects video cues, but it is important to be mindful of papers that can be noisy and picked up by the microphone. To keep the audience 


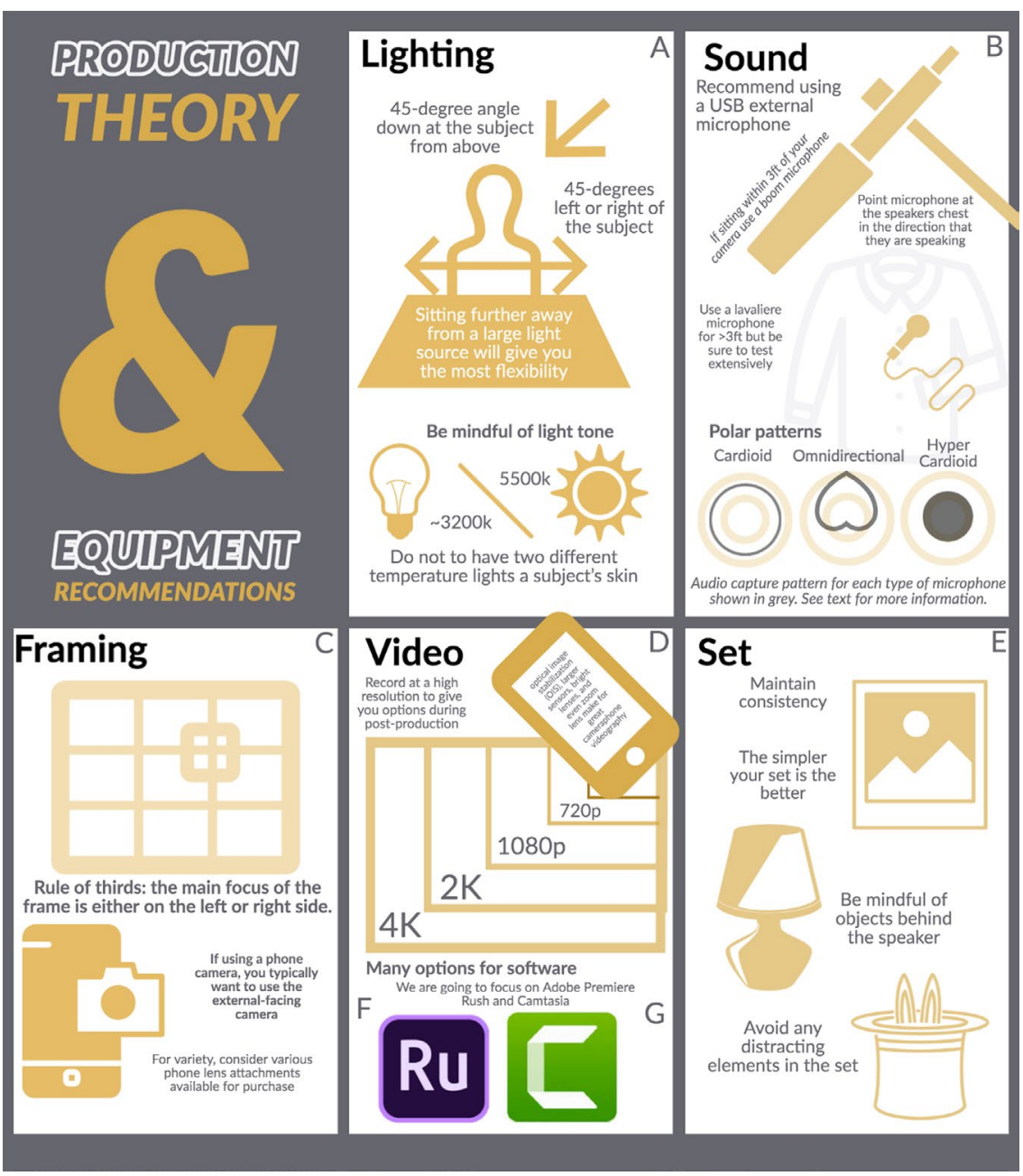

FIGURE 3. Production theory and equipment recommendations. Here, we provide an infographic that discusses the five major areas of video-production theory, including (A) lighting, (B) sound, (C) framing, (D) video, (including two video editing and recording options; (F) Adobe Rush and (G) Camtasia) and (F) set. More detailed discussion of these areas can be found in the text and on the SciVid (https://www.scivid.online/).

ate for beginners (Supplemental Video 7). When beginning production with these styles, it is first important to reflect on the purpose of the video. Knowing the purpose of the video will help determine what should be included within the frame. The challenge with filming in a classroom or doing a demonstration video is deciding whether to focus on the board, the demonstration, or the speaker. Obtaining a clear shot containing two items of focus (i.e., an object and a speaker, or a speaker and an interviewer) can be difficult with only one camera; therefore it is important to decide which object should be the primary focus and then use the rule of thirds to direct the viewer's attention. For example, if the guest answers are the focus of the video, including an over-the-shoulder shot would work well. On the other hand, if both sides of the conversation are equally important, a wide shot framing both people might be best. Another consideration for this video style is the placement of the subjects and objects. In interview style, for instance, the interviewer often will be positioned closer to the interviewee than normally comfortable. This is because the camera frame does not take into consideration the large room. Nonetheless, being mindful of the space around the subject(s) is important to make the shot feel comfortable and not crowded.

The lighting setup also depends on the video's main purpose. The light position should be based on the view of the camera. For example, if using a wide two-person shot, placing the light in the center and slightly behind the subjects can facilitate ideal lighting. If recording an over-theshoulder one-person shot, a light above the opposite shoulder of the interviewer would

engaged, be mindful of voice tone and intonations. The viewer will not see a face, so the speaker can only project energy through voice and words, placing greater importance on the audio quality in general. Therefore, microphone choice is key, and it is essential to consider the acoustics of the space. To increase the recording quality and prevent reverberations off walls, blankets or cardboard boxes can be used as low-cost options to dampen sound. Alternatively, recording in a closet or interior space might be beneficial due to the fabric "absorbing" sound reverberations. Because slides are the only visual, special attention and effort should be placed on the detail and timing of the slide points. Use the animation features in slide software to build the slide components over time, which will keep and direct the audience's attention.

\section{Room Style}

Classroom, demonstration, and interview video styles introduce increased complexity of filming and may not be appropri- generally work best. If only one camera is available, a decision will need to be made about what is most important. For all video styles in this category, the setting should be as brightly lit as possible. Overhead light adds to the overall intensity but also creates shadows on the eyes because the light is directed downward. For an at-home studio, a makeshift pop-up white bounce (white screen or board; larger is better) that is leaned against the tripod facing the speaker can reduce these shadows. Large bounces, placed as close to the subjects as possible, are ideal for these purposes.

With interviews, never underestimate how nervous a guest might be during the recording. Having everything ready when the interviewee arrives is important to allow for time to make the subject comfortable within the recording environment. Although overpromising editing capabilities should be generally avoided, let your interviewee know that editing is possible and that restarting or reframing an answer is an option. The interviewer should also avoid responding audibly to the subject's 


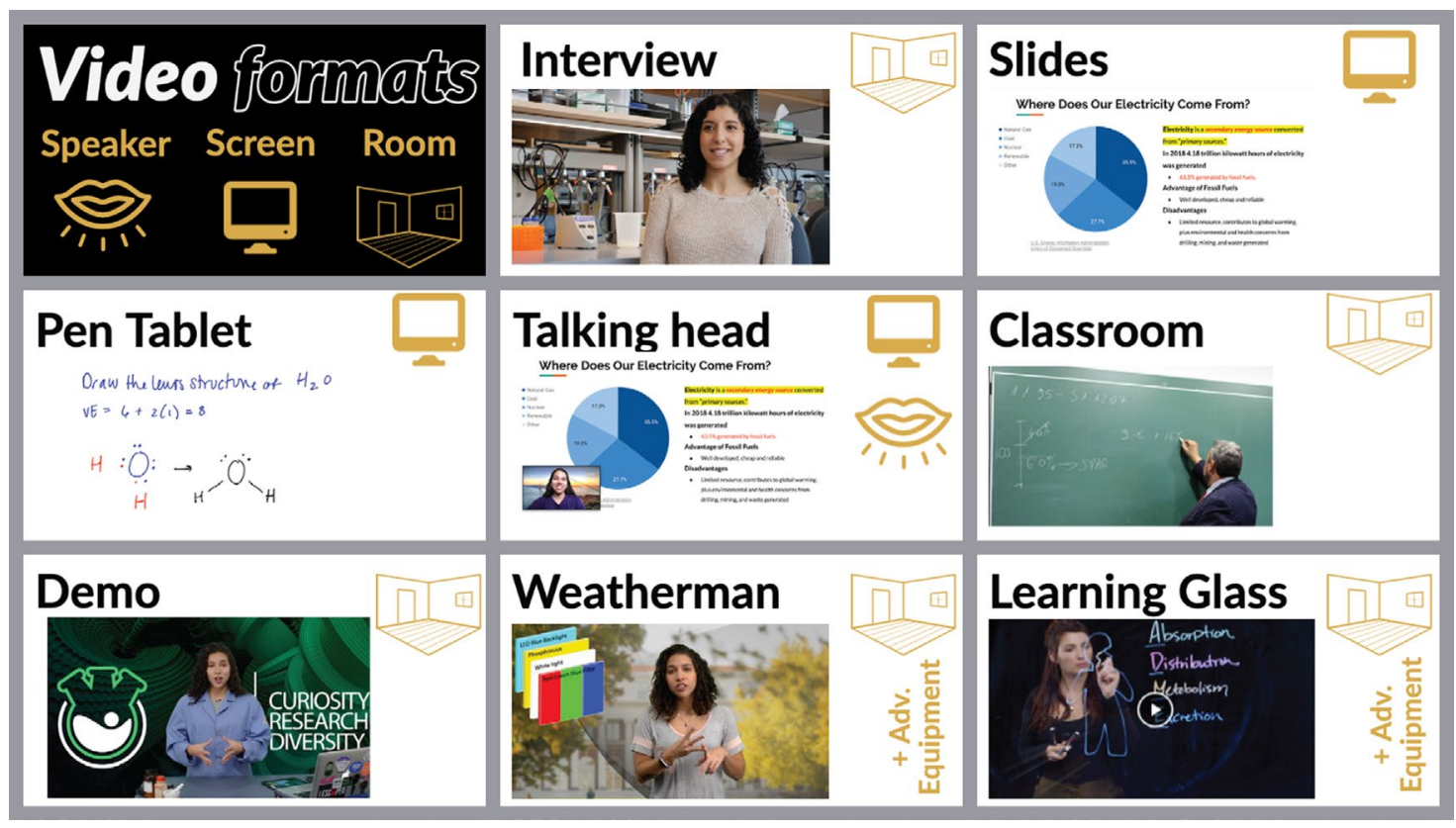

FIGURE 4. Video formats. Choe et al. (2019) evaluated eight different video styles designed to deliver standardized content in the life sciences. We have organized these formats into three main types: speaker, room, and screen. The icons for these three categories are shown in the upper right-hand corner of the video format box. The formats to which this category applies are included next to the format name on the right side. In this article, we do not discuss the weatherman or the learning class format, because additional advanced equipment is required, as indicated (+Adv. Equipment).

answers and should use head nods instead. The reason for this is not only to collect clean audio but also to avoid rushing the guest's answers.

An extra consideration for both the classroom and demonstration style is to be mindful of light exposure. Whiteboards are often reflective, and some are made of glass. Make sure the camera can capture the writing or demonstration legibly without losing text clarity to overexposure. Additionally, when testing the setup, it is important to ensure that strict framing bounds are added to prevent accidentally writing beyond the filling frame. This can be accomplished by marking the board just outside the frame, so it is clear what part of the board is captured.

\section{POSTPRODUCTION}

Postproduction encompasses all efforts occurring after the shooting or recording. Typically, this includes editing the recorded film's content and adding visual elements, music, or other sound effects. Adding these extra video elements can enhance the aesthetics of the video and cue the viewer into key concepts or major points. Before elements can be added, the captured footage's organization and a rough cut (draft) of the video should be prepared before evaluation and revision. Organizing the footage allows the educator to catalogue the recordings by the date filmed, video style, or lecture material covered. This is often referred to as "content logging," wherein a simple table is made listing the name of the file, date, and location of the footage; a brief description of the content of the video; and any other helpful notes (e.g., what might need editing; Jordan and Henderson, 1995). Videos can be organized directly into a designated folder on the computer or within the editing soft- ware. Editing software is required for postproduction, and a variety of options are available for beginners or advanced editors. Examples of editing software include Adobe Premiere, iMovie, OpenShot, Final Cut Pro X, and Camtasia. Ideally, find an editing software such as Adobe or Camtasia that is userfriendly with extensive tutorials. Once footage has been organized, import videos into the editing software of choice and begin piecing together the footage to create a rough cut of the video lecture. Refer back to the storyboard or script during this step as a guideline on how the videos should be ordered. Once the videos are compiled, additional revision processes can begin.

During this phase, the educator should revisit Mayer's (2014) multimedia principles (Supplemental Figure 1) and the video and course objectives established in preproduction (Issa et al., 2011). Consider adding visual effects such as arrows, circles, highlights, or sound effects to direct the focus of emphasizing the slide. Questions can also be inserted between scenes to lead students throughout the lesson to reach the final objective. It is essential that educator-producers avoid adding superfluous effects that do not benefit the video content's clarity. Postproduction efforts that reflect Mayer's (2014) multimedia learning principles can enhance the video's impact on learning outcomes. After going through this evaluation process, the final edited file can be exported.

\section{Using Adobe Rush or Camtasia for Editing}

The Adobe ecosystem has a rich array of tools to make videos and many other types of media. We chose Adobe Rush as an introduction to video editing, as it is a functional and approachable editing software. In Adobe Rush, it is possible to add basic 
titles, trim clip length, and move clips around with ease. It allows three layers of video and three layers of audio. If photos or videos are not being directly collected within a project, media can be added from the phone's photos, files, or cloud-based accounts. The "order item selection" command is the order by which they appear on the Adobe Rush timeline. To export from Adobe Rush, use the Sharing feature. For more advanced editing, it is advisable to download Adobe Premiere Rush onto a desktop computer. Again, there are free video-editing software options out there, but we recommend using Camtasia, as it is an editing software specifically made for educators. When it comes to editing on Camtasia, please refer to our website (www.scivid. org) for online tutorials on using this editing software.

\section{Blending Styles}

If you choose to blend two different styles in a video, it is best to record everything in one setup before moving on to the next. Completely recording each style will help build consistency and save time on setup. When organizing various styles, you should consider file organization schemes. Include a label in the first frame to visually display key pieces of information, including the project name, the video number in the series, segment number, and/or last take number in the file name. This information can be written on a piece of paper and shown at the beginning of a recording session to help during postproduction organization.

\section{File Organization and Collaboration}

During postproduction, file organization, cloud storage, and collaboration and review are critical. File organization is instrumental in streamlining postproduction and avoiding overwhelming a phone memory capacity. Furthermore, if you are blending multiple video files, it is important to know where each file is to be able to find it later. While collecting graphical and video assets, it is important to periodically back up all files. Although Adobe Premier Rush does organize the project files, be mindful of device space on the phone without an external solution. We recommend using a large external hard drive to store recordings. Cloud storage systems are a great approach that is particularly beneficial for long-term storage files.

After creating the video, determining the best way to collect and implant feedback is key. Frame.io, a software that allows frame-by-frame commenting, is an option for uploading the final video and having any number of people review and add comments in an organized format. Frame-by-frame commenting makes it very easy to know what needs to be done and where it needs to be done. Dropbox and Vimeo have also added this functionality for some of their proprietary options. If it is not possible to use this type of software, make sure that all reviewers are given clear instructions on how to interpret the time in their video player, so everyone is using the same system to provide feedback.

\section{Exporting and File Format}

The file type used to collect the video footage and the file export type are important factors that play into the video's life span. The file formats for recording and editing (e.g., MOV, RAW, MXF) are distinct from the exported video formats (MPEG/H.264, WMV, etc.). It is essential to identify the type of file formats that are produced by your recording device and the optimal exporting video file type for your intended use. While the quality of video recording continues to improve, $4 \mathrm{k}$ (3840 $\times$ 2160 ) is a useful acquisition setting that is possible when using a phone camera. Filming in 4k and exporting in 1080 pixels can be useful in some circumstances. For example, using $4 \mathrm{k}$ and exporting in 1080p can allow for a digital zooming on speaker that does not impact overall resolution. Zooming in or slightly reframing by moving the video file up or down within the frame is a great way to add dimension within the constraints of a video captured in a single recording.

\section{COPYRIGHT CONSIDERATIONS}

There are a few things to consider beyond production when creating videos. One major consideration is the use of external resources or images. Intellectual property rights protecting ownership and control over creative works or inventions are governed through copyright, trademarks, and patents. Copyright covers a wide range of artistic works. Fair use of artistic work includes things like criticism, comment, news reporting, teaching, scholarship, and research (section 107). Furthermore, section 110 (1) of Copyright Act of 1976 allows for classroom performance and display. Unfortunately, the guidelines are quite broad, and it can be unclear what may be considered fair use. Overall, there is a balance between the public's interest in open access and the interests of the copyright holder. Before using copyrighted materials in videos, conduct a fair-use analysis to determine whether it is necessary to request permission. The video-hosting platforms should also be considered, so that secondhand download and distribution are not permitted. Next, it is also important to consider the educator's intellectual property rights. Teaching in higher education, and particularly teaching in blended or online programs, is a time-intensive process. Colleges and universities are making significant investments in people (instructional designers, media educators, librarians, etc.) and digital platforms and tools in support of the teaching and learning enterprise. At the same time, every college and university would be wise to affirm principles of faculty control of material created for teaching.

\section{CONCLUSION}

The COVID-19 pandemic has challenged educators to adjust educational approaches to accommodate online teaching practices, while providing opportunities to innovate and design video content that enhances accessibility and engagement. Necessity now compels educators to explore digital media for remote teaching and learning. Although producing a video may seem like a daunting task, it can also be a creative one. Educators can be positioned as designers and producers in a way they may not have been during their previous teaching experiences. This is likely to be especially true for more experienced faculty members who may not have had any previous experience making and using videos for teaching. Rather than feeling that new approaches are being imposed, seeing video creation as a form of creative expression shifts the narrative for educators to an opportunity to learn a new skill and explore their creativity as a form of continuing professional development.

This article outlines the production process to create high-quality, effective education videos in an at-home setting using a low-budget setup. If educators are producing a large volume of complex videos, this workflow may not be appropriate, 
as advanced software, file organization, and file storage and a more advanced camera, equipment options, and workflow would be required. We have provided a simple checklist for the education video creation process across preproduction, production, and postproduction that can be customized depending of the type of video being developed (Figure 1). We have tried to address one consistent hurdle across production case studies: the effort required during preproduction is typically underestimated (Chang and Hirsch, 1994; Currie, 2003; Corbally, 2005). The preproduction process is needed to determine the type of video style based on the video's objective. Even if one style was chosen for a particular lecture, it is essential that the educator reflect on whether parts of the lecture can be better communicated through an alternative approach. Ultimately, preproduction informs the entire video-production process.

Ultimately, this article presents a process for creating digital educational resources to help guide the creation process for educators. Video is an accessible and prevalent medium for communication that can support asynchronous teaching that combats students' and educators' technical and logistical bandwidth requirements. The rising importance of video technology in biomedical science education calls for a thorough understanding of its production process by the educators who create videos to enhance video content effectiveness through design principles. The process that we describe here is one that is shared with cinema and media art. While educators explore this process, we also highly encourage educators to either work with producers or their institutions' media teams. However, if that is not possible, the educator should adopt a producer's perspective. Creative exploring of at-home instructional video production that takes these recommendations into consideration will lead to aesthetically pleasing and effective educational videos.

\section{REFERENCES}

Anderson, J. M., Aylor, M. E., \& Leonard, D. T. (2008). Instructional design dogma: Creating planned learning experiences in simulation. Journal of Critical Care, 23(4), 595-602. https://doi.org/10.1016/j.jcrc.2008.03.003

Antonietti, A., Colombo, B., \& Nuzzo, C. Di (2015). Metacognition in self-regulated multimedia learning: Integrating behavioural, psychophysiological and introspective measures. Learning, Media and Technology, 40(2), 187-209. https://doi.org/10.1080/17439884.2014.933112

Black, P., \& Wiliam, D. (1998). Assessment and classroom learning, assessment in education. Principles, Policy \& Practice, 5(1), 7-74. https://doi .org/10.1080/0969595980050102

Brame, C. J. (2016). Effective educational videos: Principles and guidelines for maximizing student learning from video content. CBE-Life Sciences Education, 15(4), es6.1-es6.6. https://doi.org/10.1187/cbe.16-03-0125

Cairncross, S., \& Mannion, M. (2001). Interactive multimedia and learning Realizing the benefits. Innovations in Education and Teaching International, 38(2), 156-164. https://doi.org/10.1080/14703290110035428

Chang, B. L., \& Hirsch, M. (1994). Videotape intervention: Producing videotapes for use in nursing practice and education. Journal of Continuing Education in Nursing, 25(6), 263-267. Retrieved February 3, 2018, from http://europepmc.org/abstract/MED/7868744

Choe, R. C., Scuric, Z., Eshkol, E., Cruser, S., Arndt, A., Cox, R., ... \& Crosbie, R. H. (2019). Student satisfaction and learning outcomes in asynchronous online lecture videos. CBE Life Sciences Education, 18(4), 1-14 https://doi.org/10.1187/cbe.18-08-0171

Clark, C., Strudler, N., \& Grove, K. (2015). Comparing asynchronous and synchronous video vs. text based discussions in an online teacher education course. Journal of Asynchronous Learning Network, 19(3), 48-69. https://doi.org/10.24059/olj.v19i3.668
Corbally, M. A. (2005). Considering video production? Lessons learned from the production of a blood pressure measurement video. Nurse Education in Practice, 5(6), 375-379. https://doi.org/10.1016/j.nepr 2005.04.003

Currie, J. (2003). A background to video production for health education American Journal of Health Education, 34(5), 302-307. https://doi.org/ 10.1080/19325037.2003.10603567

Dunn, R., \& Rundle, S. (2000). Building excellence (BE) survey: Research manual. Retrieved October 30, 2010, https://www.pcilearn.com/ AboutBESurvey.html

Farley, J., Risko, E. F., \& Kingstone, A. (2013, September). Everyday attention and lecture retention: The effects of time, fidgeting, and mind wandering. Frontiers in Psychology, 4, 1-9. https://doi.org/10.3389/fpsyg 2013.00619

Griggs, A. (n.d.). Synthesis of the Dunn and Dunn learning-style model research: Who, what, when, where, and so what? (pp. 135-140). New York: St. John's University Center for the Study of Learning and Teaching Styles.

Huang, L., Xu, M., Chen, Z., \& Liu, F. (2019). Syllabus design for teacher education MOOCs (massive open online courses): A mixed methods approach. Communications in Computer and Information Science, 1048, 156-168. https://doi.org/10.1007/978-981-13-9895 $-7 \_14$

Issa, N., Schuller, M., Santacaterina, S., Shapiro, M., Wang, E., Mayer, R. E., \& DaRosa, D. A. (2011). Applying multimedia design principles enhances learning in medical education. Medical Education, 45(8), 818-826. https://doi.org/10.1111/j.1365-2923.2011.03988.x

Jenkinson, J. (2017). The role of craft-based knowledge in the design of dynamic visualizations. In Learning from dynamic visualization: Innovations in research and application (pp. 93-117). New York, NY: Springer International Publishing. https://doi.org/10.1007/978-3-319-56204-9_5

Johnson, G., \& Hertig, S. J. (2014). A guide to the visual analysis and communication of biomolecular structural data. Nature Reviews Molecular Cell Biology, 15, 690-698.

Jordan, B., \& Henderson, A. (1995). Interaction analysis: Foundations and practice. Journal of the Learning Sciences, 4(1), 39-103. https://doi .org/10.1207/s15327809jls0401_2

Mayer, R., \& Pilegard, C. (2014). Principles for Managing Essential Processing in Multimedia Learning: Segmenting, Pre-training, and Modality Principles. In Mayer, R. (Ed.), The Cambridge Handbook of Multimedia Learning (pp. 316-344). Cambridge, UK: Cambridge University Press. https://doi .org/10.1017/CBO9781139547369.016

Mayer, R. E. (2014). Cognitive theory of multimedia learning. In Mayer, R. E. (Ed.), The Cambridge handbook of multimedia learning (2nd ed., pp. 43-71). Cambridge: Cambridge University Press. https://doi.org/10.1017/ CBO9781139547369.005

Mayer, R. E., \& Estrella, G. (2014). Benefits of emotional design in multimedia instruction. Learning and Instruction, 33, 12-18. https://doi.org/10.1016/ j.learninstruc.2014.02.004

McGarr, O. (2009). A review of podcasting in higher education: Its influence on the traditional lecture. Australasian Journal of Educational Technology, 25(3), 309-321. https://doi.org/10.14742/ajet.1136

McGill, G. G. (2017). Designing instructional science visualizations in the trenches: Where research meets production reality. In Learning from dynamic visualization: Innovations in research and application (pp. 119150). New York, NY: Springer International Publishing. https://doi org/10.1007/978-3-319-56204-9_6

Nemirovsky, R., \& Galvis, A. (2004). Facilitating grounded online interactions in video-case-based teacher professional development. Journal of Science Education and Technology, 13(1), 67-79. https://doi.org/ 10.1023/b:jOst.0000019639.06127.67

Orr, K. L., Golas, K. C., \& Yao, K. (1994). Storyboard development for interactive multimedia training. Journal of Interactive Instruction Development, 6(3), 18. https://doi.org/10.1.1.118.2147

Paas, F., \& Sweller, J. (2014). Implications of cognitive load theory for multimedia learning. In Mayer, R. E. (Ed.), The Cambridge handbook of multimedia learning (2nd ed., pp. 27-42). Cambridge: Cambridge University Press. https://doi.org/10.1017/CBO9781139547369.004 


\section{S. Castillo et al.}

Petrosino, A. J., \& Koehler, M. J. (2007). Teachers as designers: Pre- and in-service teachers authoring of anchor video as a means to professional development. In Goldman, R., Pea, Barron, B., \& Derry, S. (Eds.), Video research in the learning sciences.

Pinsky, L. E., \& Wipf, J. E. (2000). A picture is worth a thousand words: Practical use of videotape in teaching. Journal of General Internal Medicine, 15(11), 805-810. https://doi.org/10.1046/j.1525-1497.2000 $.05129 . x$

Pritchard, A. (2018). Multiple intelligences and learning styles. Ways of Learning, 106(1), 38-58. https://doi.org/10.4324/9781315460611-4
Quitadamo, I. J., \& Brown, A. (2001). Effective teaching styles and instructional design for online learning environments. http://confreg.uoregon edu/necc2001/program/

Rajadell, M., \& Garriga-Garzón, F. (2017). Educational videos: After the why, the how. Intangible Capital, 13(5). https://doi.org/10.3926/lc.1042 Retrieved August 12, 2019, from www.intangiblecapital.org/index.php/ ic/article/view/1042/668

Um, E. "Rachel”, Plass, J. L., Hayward, E. O., \& Homer, B. D. (2012). Emotional design in multimedia learning. Journal of Educational Psychology. 104(2), 485-498. https://doi.org/10.1037/a0026609 